DUKE-CGTP-05-07

hep-th/0507187

May 15, 2022

\title{
A-Model Correlators from the Coulomb Branch
}

\author{
Ilarion V. Melnikov and M. Ronen Plesser \\ Center for Geometry and Theoretical Physics \\ Box 90318 \\ Duke University \\ Durham, NC 27708-0318
}

\begin{abstract}
We compute the contribution of discrete Coulomb vacua to $A$-Model correlators in toric Gauged Linear Sigma Models. For models corresponding to a compact variety, this determines the correlators at arbitrary genus. For non-compact examples, our results imply the surprising conclusion that the quantum cohomology relations break down for a subset of the correlators.
\end{abstract}




\section{Introduction}

Topological Field Theory (TFT) is a powerful tool for studying the RG-invariant properties of non-trivial quantum field theories. A particularly important class of examples is provided by the $A$ and $B$ twists of an $\mathcal{N}=(2,2)$ SUSY Non-Linear Sigma Model (NLSM) defined on a Riemann surface $\Sigma[1,2]$. These TFTs provide rich examples of solvable quantum field theories, and they have important applications to compactification in string theory. In addition, these TFTs can be used to study enumerative geometry and refined topological invariants, such as the Gromov-Witten invariants, of many manifolds. Finally, these theories provide a natural setting for the study of mirror symmetry of Calabi-Yau manifolds [2]. Although the TFT perspective on the NLSM is immediately conceptually useful, aside from particularly tractable examples such as the classic work of Candelas et al [3] and its various generalizations [4-7], direct study of these models is difficult.

Remarkably, a large class of NLSMs, including those corresponding to Calabi-Yau manifolds constructed as hypersurfaces or complete intersections in Fano toric varieties, may be constructed as IR limits of certain $\mathcal{N}=(2,2)$ SUSY abelian gauge theories termed Gauged Linear Sigma Models (GLSMs) [8]. The RG-invariant observables of the $A$ and $B$ models may be computed in the massive theory. The $A$-model in particular has been studied extensively in $[8,9]$. It was found that many properties of the GLSM, including the correlators in the topologically twisted models, are constrained by toric geometry. For example, toric methods allow an explicit and general formulation of the instanton sum for the "toric" subset of $A$-model correlators [9]. The study of these instanton sums is enlightening: it allows for a careful definition of the monomial-divisor mirror map, and it reduces mirror symmetry to the mirror map, a non-trivial renormalization of the GLSM versus NLSM parameters. Furthermore, for a genus zero Riemann surface, the quantum restriction formula of [9] reduces the computation of topological correlators for a Calabi-Yau hypersurface to the computation of correlators on the ambient Fano toric variety. Where the mirror map is known, this allows for an explicit verification of mirror symmetry at the level of TFT. These are powerful results. Still, the instanton sums are unwieldy. In general they may be quite intricate, and simple but important properties of the correlators, like the quantum cohomology relations, seem to follow from obscure relations between intersection numbers on various toric varieties $[9,10]$. Finally, the toric methods for performing the gauge instanton sums have only been developed for genus zero correlators, and their extension to $g>0$ correlators is non-trivial. 
In this work we reduce the computation of $A$-model correlators in a wide class of GLSMs to a well-studied algebraic problem. Our result is simple to state. Consider a GLSM with a set of chiral matter $\mathcal{N}=(2,2)$ multiplets $\Phi^{i}, i=1, \ldots, n$, charged under a gauge group $[\mathrm{U}(1)]^{r}$ with charges $Q_{i}^{a}, a=1, \ldots, r$, and zero superpotential for the matter fields. Such models will be referred to as toric GLSMs. Upon twisting, the local $A$-model observables are found to be functions of the $\sigma_{a}$, the bosonic super-partners of the gauge fields. The most general $A$-model correlator on $\Sigma_{g}$, a Riemann surface of genus $g$, may be obtained from linear combinations of $\left\langle\sigma_{a_{1}}\left(z_{1}\right) \cdots \sigma_{a_{s}}\left(z_{s}\right)\right\rangle_{g}$ and derivatives of these with respect to the GLSM parameters. Since this is a TFT computation, the correlator is independent of the generic points $z_{1}, \ldots, z_{s}$ on $\Sigma_{g}$. Following a standard notation, we will denote these correlators by $\langle F(\sigma)\rangle_{g}$, where $F(\sigma)$ is to be understood as a power series in $\sigma_{a_{k}}\left(z_{k}\right)$ with a generic choice of the $z_{k}$. In a sense made more precise below, "most" toric GLSMs possess a region of parameter space where the theory has a number of discrete Coulomb vacua determined as solutions to the equations of motion for a certain effective twisted superpotential $\widetilde{W}_{\text {eff }}(\sigma)$. This class includes all GLSMs corresponding to compact toric varieties. For these compact, toric GLSMs there is an additional simplification: there exists a region of the parameter space where these discrete Coulomb vacua are the only vacua. In this case, the $A$-model correlators at genus $g$ are given by

$$
\langle F(\sigma)\rangle_{g}=\sum_{\hat{\sigma} \mid d W_{\mathrm{eff}}(\hat{\sigma})=0} \tilde{H}(\hat{\sigma})^{g-1} F(\hat{\sigma})
$$

where $\widetilde{H}(\sigma)=H \prod_{i=1}^{n} \xi_{i}, H$ is the Hessian of $\widetilde{W}_{\text {eff }}$, and $\xi_{i}=\sum_{a} Q_{i}^{a} \sigma_{a}$. The equations of motion that follow from $d \widetilde{W}_{\text {eff }}=0$ are polynomial in the $\sigma_{a}$, so the computation of $A$-model correlators is now reduced to an algebraic problem.

This form is convenient for obtaining explicit correlators in many toric examples. Furthermore, by the use of the quantum restriction formula of [9], it becomes a useful tool to compute genus zero $A$-model correlators on GLSMs corresponding to Calabi-Yau surfaces. In addition, eqn.(11) manifestly satisfies the quantum cohomology relations, and, as we will see in more detail below, it is a useful probe for the physics of the Coulomb branch of the GLSM.

Since we have not coupled the TFT to topological gravity, our result is of limited use for the computation of general Gromov-Witten invariants-factorization of the correlators 
implies that our higher genus results do not generate "new" invariants for $g>0$. However, we believe that for the purposes of enumerative geometry, eqn.(1) is a neat packaging of the requisite combinatorics. Essentially, while it is true that the $g>0$ correlators may be obtained by factorization from the $g=0$ correlators, if one interested in explicit numbers, eqn.(11) may eliminate much algebraic suffering.

It is no accident that the form we find is reminiscent of the correlators in topological Landau-Ginzburg models studied by Vafa [11]. In fact, our analysis is a simple extension of those techniques to include the zero modes of the matter fields. These additional zero modes are the source of the factor of $\prod_{i} \xi_{i}$ in our expression. We will discuss this further below.

While eqn.(11) computes the correlators in compact toric GLSMs, in non-compact toric GLSMs the above expression is only a part of the story. In general, only a subset of the solutions to $d \widetilde{W}_{\text {eff }}=0$ correspond to Coulomb vacua, and eqn.(11) provides the correct measure for the contributions to the correlators due to these vacua. In addition, other, nonCoulomb vacua may also contribute, and these contributions may invalidate the quantum cohomology relations.

The rest of this note is organized as follows. In section 2 we briefly review the toric GLSM and the corresponding $A$-model, and we take care to distinguish the GLSM phases according to the properties of the Coulomb vacua. In section [3] we prove eqn.(11) by studying the $A$ model localization onto the Coulomb vacua. We provide some applications of the result to compact toric GLSMs in section 4. In section 5 we turn to a study of a non-compact example, and we conclude with a discussion in section [6.

\section{A GLSM Overview}

\subsection{Some Superspace Details}

The GLSM is a $d=2$ abelian gauge theory with $\mathcal{N}=(2,2)$ supersymmetry. The field content is neatly summarized in terms of $\mathcal{N}=(2,2)$ multiplets. The matter fields belong to chiral multiplets $\Phi^{i}=\left(\phi^{i}, \psi_{ \pm}^{i}, \bar{\psi}_{ \pm}^{i}, F^{i}\right)$, with $\phi^{i}$ a complex scalar, $\psi_{ \pm}^{i}$ left/right-moving Weyl fermions, $F^{i}$ a complex auxiliary field, and $i=1, \ldots, n$. These fields are charged under the gauge group $G=[\mathrm{U}(1)]^{r}$ with integral charges $Q_{i}^{a}, a=1, \ldots, r$. The gauge fields reside in real vector supermultiplets $V_{a}$, and the gauge-invariant field-strengths are to be found in twisted chiral multiplets $\Sigma_{a}=\left(\sigma_{a}, \lambda_{ \pm, a}, \bar{\lambda}_{ \pm, a}, D_{a}-i f_{01, a}\right)$, where $\sigma_{a}$ is a complex scalar, 
$\lambda_{ \pm, a}$ are left/right-moving Weyl fermions, $D_{a}$ is a real auxiliary field, and $f_{01, a}$ is the abelian gauge field-strength.

We define the GLSM at a scale $\mu$ by a Lagrange density $\mathscr{L}^{\mu}$ given by a sum of two terms, the Kähler term $\mathscr{L}_{K}^{\mu}$ and the twisted superpotential term $\mathscr{L}_{\widetilde{W}}^{\mu}$. We take the Kähler term to be

$$
\mathscr{L}_{K}^{\mu}=\int d^{4} \theta\left(-\frac{1}{4} \sum_{i=1}^{n} \bar{\Phi}^{i} \exp \left(2 \sum_{a=1}^{r} Q_{i}^{a} V_{a}\right) \Phi^{i}+\frac{1}{4 \mu^{2} g(\mu)^{2}} \sum_{a=1}^{r} \bar{\Sigma}_{a} \Sigma_{a}\right),
$$

where $g(\mu)$ is the dimensionless coupling of the gauge theory. The tree-level twisted superpotential is given by

$$
\mathscr{L}_{\widetilde{W}}^{\mu}=\left[-\frac{i}{2 \sqrt{2}} \int d \theta^{+} d \bar{\theta}^{-} \sum_{a=1}^{r} \Sigma_{a} \tau^{a}(\mu)\right]+\text { c.c. }
$$

The $\tau^{a}(\mu)=i r^{a}(\mu)+\frac{\theta^{a}}{2 \pi}$ are the parameters of the model. Each $\tau^{a}$ is a combination of a Fayet-Iliopoulos (F-I) term $r^{a}$ and a $\theta$-angle $\theta^{a}$. It is useful to define single-valued parameters $q_{a}=e^{2 \pi i \tau^{a}}$. For generic values of these parameters the moduli space of classical vacua of the GLSM so defined is a toric variety. The GLSM may be generalized by including a superpotential $W(\Phi)$ which serves to restrict the moduli space to a hypersurface or a complete intersection in the ambient toric variety. We will restrict attention to toric GLSMs, those with $W(\Phi)=0$.

\subsection{Basic GLSM Properties}

Let us begin with a brief review of the Higgs vacua of the GLSM. ${ }^{1}$ This material is well known, and we refer the reader to [8,9] for further details. The classical moduli space of a toric GLSM is obtained by solving the $D$-terms modulo the gauge group as functions of the F-I parameters $r^{a}$. One finds that there exists a cone $\mathcal{K}_{c} \subseteq \mathbb{R}^{r}$ where the space of solutions to the $D$-terms is non-empty. ${ }^{2}$ For generic values of the $r^{a} \in \mathcal{K}_{c}$, the gauge group is completely broken, the $\sigma_{a}$ are massive, and the moduli space is a toric variety of complex dimension $d=n-r$. The geometric properties of this toric variety vary smoothly with the $r^{a}$ away

\footnotetext{
${ }^{1}$ There are no photons and no Higgs mechanism in two dimensions, and a description based on Higgs vacua is only valid at weak coupling. Fortunately, this is just where we will use it, and so we will ignore this subtlety in what follows.

${ }^{2}$ The D-term equations are $D^{a}=\sum_{i} Q_{i}^{a}\left|\phi^{i}\right|^{2}-r^{a}$, whence it follows that $\mathcal{K}_{c}$ is indeed a cone, the space positively generated by the $n$ vectors $\mathbf{Q}_{i} \in \mathbb{R}^{r}$.
} 
from co-dimension one sub-cones of $\overline{\mathcal{K}_{c}}$, where the gauge group is un-Higgsed and some or all of the $\sigma_{a}$ become massless. These boundaries subdivide $\mathcal{K}_{c}$ into a set of cones $\mathcal{K}_{V}$, indexed by a set of birationally equivalent toric varieties. There is a natural association between the varieties and the cones: for $r^{a} \in \mathcal{K}_{V}$ the moduli space of the GLSM's classical vacua is the variety $V$. The cones $\mathcal{K}_{V}$ are termed phases of the GLSM. By choosing the F-I parameters deep in the interior of any such phase, we obtain a weakly coupled theory whose low energy theory is that of a NLSM with target-space $V$. For reasons that will become clear below, we will also refer to the complement of $\mathcal{K}_{c}$ as a phase.

As the F-I terms are tuned to approach a lower dimensional face of $\mathcal{K}_{V}$, the low energy description seems to break down as $V$ becomes singular, or equivalently, there appear new massless degrees of freedom corresponding to an un-Higgsed subgroup of the gauge group. Quantum effects lift the corresponding singularities when the un-Higgsed gauge group satisfies $\sum_{i} Q_{i} \neq 0$, and even for gauge groups with $\sum_{i} Q_{i}=0$, the singularities are lifted for generic values of the corresponding $\theta$ angle. Thus, all phases are smoothly connected, and the low-energy NLSM description is smooth away from a complex co-dimension one subvariety in the space of the $q_{a}$-the singular locus. Of course, from the point of view of the GLSM there is no real singularity on the singular locus. However, we do expect that the theory is strongly coupled on the singular locus, and strong coupling effects may invalidate results based on the weakly coupled description.

In addition to the Higgs vacua, the GLSM possesses Coulomb vacua. These are obtained when some of the $\sigma_{a}$ acquire non-zero expectation values and give masses to some or all of the matter fields. Integrating out these massive $\Phi^{i}$ multiplets leads to an effective interaction for the $\Sigma_{a}$ fields, which can be expressed in terms of an effective twisted superpotential $\widetilde{W}_{\text {eff }}(\Sigma)[8,9]$. The solutions to $d \widetilde{W}_{\text {eff }}(\sigma)=0$ are continuous if $\sum_{i} Q_{i}^{a}=0$ for all $\sigma_{a}$ with non-zero expectation values, and they are discrete otherwise. The former exist only on the singular locus of the model, while the latter vary smoothly with the parameters. When all of the matter fields are massive, $\widetilde{W}_{\text {eff }}(\Sigma)$ is given by ${ }^{3}$

$$
\widetilde{W}_{\text {eff }}=\sum_{a=1}^{r} \Sigma_{a} \log \left[\prod_{i=1}^{n}\left(\frac{1}{\exp (1) \mu} \sum_{b=1}^{r} Q_{i}^{b} \Sigma_{b}\right)^{Q_{i}^{a}} / q_{a}\right] .
$$

\footnotetext{
${ }^{3}$ We have left off a conventional over-all factor of $-\frac{1}{4 \pi \sqrt{2}}$. As far as our results are concerned, this factor can be absorbed in the definition of the string coupling.
} 
The vacua corresponding to $d \widetilde{W}_{\text {eff }}=0$ will occupy us for most of this note. For future reference, we give the equations of motion which follow from $d \widetilde{W}_{\text {eff }}=0$ :

$$
\prod_{i \mid Q_{i}^{a}>0}\left(\frac{\xi_{i}}{\mu}\right)^{Q_{i}^{a}}=q_{a} \prod_{i \mid Q_{i}^{a}<0}\left(\frac{\xi_{i}}{\mu}\right)^{-Q_{i}^{a}}, \quad a=1, \ldots, r
$$

where $\xi_{i}=\sum_{a} Q_{i}^{a} \sigma_{a}$. We will also have use for

$$
\mathscr{H}^{a b}:=\frac{\partial^{2} \widetilde{W}_{\text {eff }}}{\partial \sigma_{a} \partial \sigma_{b}}=\sum_{i} \frac{Q_{i}^{a} Q_{i}^{b}}{\xi_{i}}
$$

Of course, the Hessian of $\widetilde{W}_{\text {eff }}$ is given by $H=\operatorname{det} \mathscr{H}$.

The Coulomb vacua are derived by integrating out massive matter fields, and thus are only reliable in the regions of the parameter space where these fields are indeed massive. In principle, this may depend on the renormalization of the Kähler terms, but at least in the weak coupling regimes (i.e deep in the interior of some $\mathcal{K}_{V}$ ) one may discern which Coulomb vacua are reliable. At weak coupling, the $\phi$ mass term has the contribution $2 \sum_{i, a, b}\left|\phi^{i}\right|^{2} Q_{i}^{a} Q_{i}^{b} \sigma_{a} \bar{\sigma}_{b}$, so that a critical point of $\widetilde{W}_{\text {eff }}$ is not reliable if all the $\sigma_{a}$ are small.

When do these discrete Coulomb vacua arise? By examining the equations of motion in eqn.(15) it is clear that these are homogeneous in the $\sigma_{a}$ whenever $\sum_{i} Q_{i}^{a}=0$ for all $a$, leading to a continuous set of solutions for the $\sigma_{a}$. We have not shown it, but it seems likely that if $\operatorname{rank}(Q)=r$ then this homogeneity is the only way to obtain a continuum of solutions. So, we expect that $\widetilde{W}_{\text {eff }}$ describes discrete Coulomb vacua whenever $\sum_{i} Q_{i}^{a} \neq 0$ for some $a$. It is always possible to choose a basis for the action of the gauge group so that $Q_{i}^{a}$ satisfy $\sum_{i} Q_{i}^{a}=0$ for $a>1$. We will work in this basis, taking $\Delta=\sum_{i} Q_{i}^{1}$. The condition for $\widetilde{W}_{\text {eff }}$ to describe discrete Coulomb vacua is then just $\Delta \neq 0$. When $\Delta=0$, the continuous solutions to $d \widetilde{W}_{\text {eff }}=0$ emerge on the principal component of the singular locus [9].

The $\widetilde{W}_{\text {eff }}$ above describes the vacua where all of the $\Phi^{i}$ are massive. Of course, there may also be Coulomb-Higgs vacua, where the gauge group is partially Higgsed. Just as the Coulomb vacua described by $\widetilde{W}_{\text {eff }}$, these may be labelled according to the space of $\sigma$ vevs as either continuous or discrete. The former are found on various non-principal components of the singular locus of the model [9], while the latter, like their discrete Coulomb cousins, may be found in various phases. We will not study the discrete Coulomb-Higgs vacua in this note. However, when analyzing a particular phase one should be careful to check that the 
results are not invalidated by the presence of these vacua.

We will find it useful to characterize the phases of the GLSM by the types of vacua found at weak coupling. Whenever a phase does not have any discrete Coulomb-Higgs vacua, we will refer to it as:

- a Geometric Phase if its weak coupling limit has no reliable Coulomb vacua, and the vacua are purely Higgs;

- a Non-Geometric Phase if the situation is reversed and there are no Higgs vacua;

- a Mixed Phase if both Coulomb and Higgs vacua are present at weak coupling.

These distinctions are important. For example, in a model with a Non-Geometric Phase, eqn.(11) yields the correlators at any genus, while in a model with a Geometric Phase, we may be able to compute the genus zero correlators by gauge instanton sums. As we will see below, in a Mixed Phase the correlators may be obtained by simply adding the Higgs and Coulomb contributions. Note that a Non-Geometric Phase may only exist outside of $\mathcal{K}_{c}$, so

it is only if $\mathcal{K}_{c} \neq \mathbb{R}^{r}$, that our strongest results hold. Happily, this holds for compact toric GLSMs.

\section{$3 \quad A$-Model Localization on the Discrete Coulomb Branch}

\subsection{Twisting and Localization: Generalities}

The topological twisting of an $\mathcal{N}=(2,2)$ theory may be accomplished by shifting the spin connection on the world-sheet by the (ultraviolet) $R$-symmetry of the model. In effect, this produces a new theory by modifying the spins of the fields. Let us now point out some basic consequences of this twisting in the context of the GLSM. The twisted theory possesses a world-sheet anti-commuting scalar operator $\mathcal{Q}$ which can be used to project the theory onto the $\mathcal{Q}$-cohomology. In the GLSM, this leads to topological observables parametrized by powers of the $\sigma_{a}$. Another consequence of the twisting is that the path integral localizes onto the $\mathcal{Q}$-invariant configurations - the SUSY vacua of the untwisted theory. This property of localization $[1,2,12-14]$ plays a crucial role in the study of TFT. Roughly, this is the statement that the path integral will localize onto the vacua of the theory, and, under certain conditions, the contribution of a particular vacuum may be computed semi-classically. This still leaves a difficult problem, especially when quantum vacua are involved. Of course, this is the case 
when we wish to study the correlators in a Mixed or Non-Geometric Phases of the GLSM. Fortunately, in that case the vacua are controlled by $\widetilde{W}_{\mathrm{eff}}(\Sigma)$, a quantity determined by holomorphy and 't Hooft anomaly matching.

A further simplification makes the TFT computations tractable: since the singular locus is a complex co-dimension one variety in the space of the $q_{a}$, we expect that we should be able to compute correlators at weak coupling in any phase, and then unambiguously obtain the result for generic $q_{a}$ by analytic continuation. Indeed, this has been demonstrated for the Geometric Phases in [9]. As we will see below, the result also holds in more general situations involving the Coulomb vacua.

\section{$3.2 \quad A$-Twist Details}

On a Euclidean signature world-sheet $\Sigma_{g}$ the spin connection may be thought of as a U(1) connection, and twisting amounts to shifting the Lorentz $\mathrm{U}(1)$ charges of the fields by a combination of the $R$-charges. In the toric GLSM the classical $\mathrm{U}(1)_{+} \times \mathrm{U}(1)_{-} R$-symmetry group leaves the superfields $\Phi^{i}, V_{a}$ invariant while acting on the $\theta^{ \pm}$with charges $Q_{ \pm}\left(\theta^{ \pm}\right)=$ $+1, Q_{\mp}\left(\theta^{ \pm}\right)=0$. All other charges are determined by this choice, and, in particular, $Q_{ \pm}\left(\sigma_{a}\right)= \pm 1$. When $\Delta \neq 0$, this classical $R$-symmetry suffers from an anomaly in the presence of gauge instantons. That means that only the vector combination may be used to obtain a consistent twisted theory. This is the $A$-model, obtained by twisting with $Q_{V}=$ $\frac{1}{2}\left(Q_{+}+Q_{-}\right)$. If we designate the Lorentz charges of the fields by $Q_{L}$, the new Lorentz charges are $Q_{L}^{\prime}=Q_{L}-Q_{V}$. Applying this to the fields of the GLSM we find that the $\phi^{i}, \sigma_{a}$ remain world-sheet scalars, but the spins of the fermions are shifted. The $\psi_{+}, \bar{\psi}_{-}, \lambda_{-}, \bar{\lambda}_{+}$become world-sheet one-forms, while $\psi_{-}, \bar{\psi}_{+}, \lambda_{+}, \bar{\lambda}_{-}$become world-sheet scalars [2]:

$$
\begin{aligned}
& \psi_{+} \rightarrow \psi_{z} \lambda_{+} \rightarrow \eta \\
& \psi_{-} \rightarrow \chi \lambda_{-} \rightarrow \rho_{\bar{z}} \\
& \bar{\psi}_{+} \rightarrow \bar{\chi} \bar{\lambda}_{+} \rightarrow \bar{\rho}_{z} \\
& \bar{\psi}_{-} \rightarrow \bar{\psi}_{\bar{z}} \bar{\lambda}_{-} \rightarrow \bar{\eta} .
\end{aligned}
$$

\subsection{Localization}

We are interested in computing expectation values of the form $\langle F(\sigma)\rangle_{g}$ in the twisted theory. As described above, to perform these computations we deform the model to weak coupling, 
where we have a reasonable handle on the vacua of the theory. Since the TFT path integral localizes onto the vacua, we may compute the correlators by summing contributions from the vacua. Let us suppose we are working in a phase of the GLSM without discrete CoulombHiggs vacua. At weak coupling, the path integral then receives contributions from the Higgs vacua - the gauge instantons, and the discrete Coulomb vacua:

$$
Z=Z^{\text {Higgs }}+Z^{\text {Coulomb }}
$$

At $g=0$, the contribution from the gauge instantons may be determined by the methods of Morrison and Plesser [9], and we will now show how to compute $Z^{\text {Coulomb }}$ at any genus.

Since we wish to compute $\langle F(\sigma)\rangle_{g}$, we may first perform the integration over the $\Phi^{i}$ multiplets. As we have argued above, in the untwisted theory this leads to a factor of $\exp \left(-\int d^{2} z \mathscr{L}_{\widetilde{W}_{\text {eff }}}\right)$ in the path integral over the $\Sigma_{a}$ multiplets. When we perform this integration in the twisted theory, we find a similar result, but we must be careful of one subtlety: the zero modes of the $\Phi^{i}$ multiplets. In the untwisted theory the $\psi_{ \pm}, \bar{\psi}_{ \pm}$had no zero modes, and, aside from the factor of $\exp \left(-\int d^{2} z \mathscr{L}_{\widetilde{W}_{\text {eff }}}\right)$ and a deformation of the (irrelevant) Kähler term of the $\Sigma_{a}$, the one-loop fermion determinants cancelled the bosonic ones as a consequence of the $\mathcal{N}=(2,2)$ SUSY. In the twisted theory, while the non-zero modes of the $\Phi^{i}$ multiplets continue to be paired up just as in the untwisted theory, the zero modes of the fermions no longer pair up with the $\phi^{i}$ zero modes. To deal with this subtlety, we will separate out the integral over the $\Phi^{i}$ zero modes. Of course, integrating out the non-zero modes will still lead to the factor of $\exp \left(-\int d^{2} z \mathscr{L}_{\widetilde{W}_{\text {eff }}}\right)$.

We are now in a position to apply the standard localization arguments to the Coulomb vacua. The corresponding $\mathcal{Q}$-invariant configurations are a subset of the field configurations

$$
\phi^{i}=0, \quad f_{a}=0, \quad \partial_{z} \sigma_{a}=\partial_{\bar{z}} \sigma_{a}=0, \quad d \widetilde{W}_{\mathrm{eff}}=0 .
$$

A solution to $d \widetilde{W}_{\text {eff }}(\sigma)=0$ does not necessarily correspond to a Coulomb vacuum, and at generic $q_{a}$ it is difficult to determine which of the solutions to $d \widetilde{W}_{\text {eff }}(\sigma)=0$ are reliable. However, at weak coupling, i.e. deep in the interior of a phase, we may answer this question unambiguously: a $\sigma$ vacuum is trustworthy only if in the weak coupling limit the corresponding $\left|\sigma_{a}\right|$ grow in such a way that all of the $\Phi^{i}$ multiplets may be consistently integrated out. 
We will label these reliable vacua by $\hat{\sigma}$. The contribution to the path integral is then

$$
Z^{\text {Coulomb }}=\sum_{\hat{\sigma}} Z(\hat{\sigma})
$$

and we can compute $Z(\hat{\sigma})$ at weak coupling by expanding in fluctuations about the $\hat{\sigma}$ vacuum. Integration over the massive modes of the $\Sigma$ multiplets leads to determinants that exactly cancel between the bosons and fermions (this familiar fact may be traced back to the primordial $\mathcal{N}=(2,2)$ SUSY), and we are left with an integral over the zero-modes, which factorizes into an integral over the $\Phi$ fluctuations and an integral over $\Sigma$ fluctuations:

$Z(\hat{\sigma})=\int[D \Phi] \int[D \Sigma] \exp \left(-S_{\Phi}-S_{\Sigma}\right)=\int[D \Phi] \exp \left(-S_{\Phi}\right) \int[D \Sigma] \exp \left(-S_{\Sigma}\right)=Z_{\Phi}(\hat{\sigma}) Z_{\Sigma}(\hat{\sigma})$

The terms in the action are given by

$$
\begin{aligned}
-S_{\Phi} & =\sum_{i}\left\{V_{\Sigma}\left[-2\left|\xi_{i}\right|^{2}\left|\phi^{i}\right|^{2}+\sqrt{2} \chi^{i} \bar{\xi}_{i} \bar{\chi}^{i}\right]+\sqrt{2} \int_{\Sigma_{g}} \psi_{z}^{i} \xi_{i} \wedge \bar{\psi}_{\bar{z}}^{i}\right\}, \\
-S_{\Sigma} & =\sum_{a, b}\left\{V_{\Sigma}\left[-4 \mu^{2} \bar{\sigma}_{a}\left(\mathscr{H}^{\dagger} \mathscr{H}\right)^{a b} \sigma_{b}+\bar{\eta}_{a} 2 \overline{\mathscr{H}}^{a b} \eta_{b}\right]+\int_{\Sigma_{g}} \bar{\rho}_{z, a} 2 \mathscr{H}^{a b} \wedge \rho_{\bar{z}, b}\right\},
\end{aligned}
$$

where $V_{\Sigma}$ is the volume of $\Sigma_{g}$, and $\xi_{i}$ and $\mathscr{H}^{a b}$ - the latter defined in eqn.(6) - are to be evaluated at $\sigma=\hat{\sigma}$. We can now evaluate the Gaussian integrals. For $Z_{\Phi}$ we find

$$
\begin{gathered}
Z_{\Phi}=Z_{\phi} Z_{\chi} Z_{\psi}, \\
Z_{\phi}^{-1}=V_{\Sigma}^{n} \prod_{i}\left|\xi_{i}\right|^{2}, \quad Z_{\chi}=V_{\Sigma}^{n} \prod_{i} \bar{\xi}_{i}, \quad Z_{\psi}=\prod_{i} \xi_{i}^{g} .
\end{gathered}
$$

We have counted the 1 zero mode of $\chi$ (there is just one constant function on a compact $\Sigma_{g}$ ) and the $g$ zero modes of the $\psi_{z}^{i}$ (there are $g$ holomorphic one-forms on $\Sigma_{g}$ ). Similarly,

$$
Z_{\Sigma}=Z_{\sigma} Z_{\eta} Z_{\rho}
$$


with

$$
Z_{\sigma}^{-1}=V_{\Sigma}^{r}|H|^{2}, \quad Z_{\eta}=V_{\Sigma}^{r} \bar{H}, \quad Z_{\rho}=H^{g},
$$

where $H=\operatorname{det} \mathscr{H}$. Putting it all together, we find the measure in eqn.(11).

The careful reader will note that we have ignored any subtleties associated to gauge invariance. This simplification follows because the computations are performed on the Coulomb branch, where the condition $f_{a}=0$ and the Riemann-Roch theorem ensure that our computation of $Z_{\Phi}$ is correct. We have also neglected various constants which may be absorbed into an overall normalization of the correlators or a re-definition of the string coupling constant. Furthermore, the sign of the fermion integration measure has been chosen to match results from the Higgs Phase computations at genus zero.

In what follows, we will work in units of the scale $\mu$. This scale plays an important role in the untwisted theory, but upon twisting it becomes superfluous, essentially because the TFT is a theory at zero energy. The scale can be important if one wants to make connections with the untwisted theory, in which case it is easy to restore in our formulas.

\section{A Few Applications to Compact Toric GLSMs}

As discussed above, we expect that whenever $\mathcal{K}_{c} \nsucceq \mathbb{R}^{r}$, eqn. (11) directly gives the correlators at arbitrary genus. This makes it useful for elucidating various properties of the compact toric GLSM $A$-model correlators, as well as actual computations. We illustrate this in this section.

\subsection{Some Properties of the Correlators}

We can easily demonstrate some important properties of these GLSM $A$-model correlators from the explicit form. Perhaps the simplest observation is that the result presents the correlators as a sum over all the solutions to a system of polynomial equations with finitely many common zeroes. This finite sum has a natural expansion in terms of symmetric functions, and, thus, it is clear that the correlators are meromorphic functions of the $q_{a}$. This is not obvious from the form of the instanton sum in a Geometric Phase. Another equally simple but important observation is that the quantum cohomology relations, which are just the equations of motion in eqn.(5) considered as operator relations, obviously hold. This should also be compared with the Geometric phase computation, where this is a non-trivial 
combinatorics result $[9,10]$. Below, we give a few more technical observations.

\subsubsection{TFT Factorization}

The $A$-twist reduces the Hilbert space of the GLSM to a vector space of dimension $N_{v}$, where $N_{v}$ is the number of discrete Coulomb vacua. The operators $\sigma_{a}$ are now simply $N_{v} \times N_{v}$ matrices, and correlators are obtained by taking a matrix trace:

$$
\langle F(\sigma)\rangle_{g}=\operatorname{Tr}\left[F(\sigma)(\widetilde{H}(\sigma))^{g-1}\right]
$$

These correlators are easily shown to satisfy the factorization axioms of topological field theory. These axioms state that if we choose a complete basis of states $|i\rangle$, and the corresponding operators $\phi_{i}$ have the metric $\eta_{i j}=\left\langle\phi_{i} \phi_{j}\right\rangle_{0}$, with inverse $\eta^{i j}$, then

1. if $F(\sigma)=f_{1}(\sigma) f_{2}(\sigma)$, then

$$
\langle F(\sigma)\rangle_{g}=\sum_{i j}\left\langle f_{1}(\sigma) \phi_{i}\right\rangle_{g^{\prime}} \eta^{i j}\left\langle\phi_{j} f_{2}(\sigma)\right\rangle_{g-g^{\prime}}
$$

and

2. for any $F(\sigma)$

$$
\langle F(\sigma)\rangle_{g}=\sum_{i j} \eta^{i j}\left\langle\phi_{i} \phi_{j} F(\sigma)\right\rangle_{g-1}
$$

These properties are apparent in a basis of states corresponding to the $N_{v} \sigma$-vacua. The state operator correspondence is $|i\rangle \leftrightarrow \phi_{i}=\delta_{\sigma, \sigma_{i}}$, where $\sigma_{i}$ is the value of $\sigma$ in the $i$-th vacuum. In this basis the operator $\sigma$ is diagonal, and $\eta^{i j}=\widetilde{H}\left(\sigma_{i}\right) \delta^{i j}$. Factorization follows immediately. Thus, as expected, any genus correlator may be obtained from the $g=0$ results.

\subsubsection{The Ghost Number Selection Rule}

These $A$-model correlators obey a simple selection rule. Working in the basis where

$$
\sum_{i} Q_{i}^{1}=\Delta \text { and } \sum_{i} Q_{i}^{a}=0 \text { for } a>1
$$

we may write the $\sigma$ equations of motion in the form $\sigma_{a}=\omega_{a} \sigma_{1}$ for $a>2$, where $\omega_{a}$ are now determined by solving $r-1$ polynomial equations, and $\sigma_{1}$ satisfies $\sigma_{1}^{\Delta}=q_{1} s(\omega)$ for some 
$s(\omega)$. Thus, the sum over the vacua includes a sum over the $\Delta$-th roots of unity. Since $\widetilde{H}$ has degree $d=n-r$, if $F(\sigma)$ has degree $s$, then $\langle F(\sigma)\rangle_{g}$ is non-zero only if $s+d(g-1)=m \Delta$ for some integer $m$, in which case $\langle F(\sigma)\rangle_{g} \sim q_{1}^{m}$. This selection rule is just the ghost number selection rule familiar from TFTs in general and GLSMs in particular.

\subsubsection{The All Genus Correlation Function}

Although factorization makes this exercise purely one of convenience, we can easily sum over the genera to obtain

$$
\langle F(\sigma)\rangle=\sum_{g \geq 0} \lambda^{2 g-2}\langle F(\sigma)\rangle_{g}=\operatorname{Tr}\left[\frac{F(\sigma)}{\lambda^{2} \widetilde{H}} \frac{1}{1-\lambda^{2} \widetilde{H}}\right]
$$

From the selection rule above it follows that if $F(\sigma)$ has degree $s$ then

$$
\langle F(\sigma)\rangle=q_{1}^{s / \Delta} f\left(\left(q_{1}^{d} \lambda^{2 \Delta}\right)^{1 / g c d(d, \Delta)}\right)
$$

\subsubsection{The Quantum Restriction Formula}

Given a Calabi-Yau hypersurface in a toric variety $V$, there exists a simple method for obtaining the "toric" subset [15] of the $g=0 A$-model correlators for the Calabi-Yau model. These correlators can be computed by the quantum restriction formula of [9], which expresses

a hypersurface toric $A$-model correlator, denoted by $\langle\langle F(\sigma)\rangle$, to a sum over the $A$-model correlators for $V$ :

$$
\langle\langle F(\sigma)\rangle\rangle_{g=0}=\left\langle F(\sigma) \frac{-K}{1-K}\right\rangle_{g=0}
$$

where $-K$ is the operator corresponding to the anti-canonical divisor on $V$, given by $-K=$ $\sum_{i} \xi_{i}$. Using our form of the correlators on $V$, it follows that

$$
\langle\langle F(\sigma)\rangle\rangle_{g=0}=\operatorname{Tr}\left[\frac{F(\sigma)}{\widetilde{H}} \frac{-K}{1-K}\right] .
$$

\subsection{Two Examples}

In this section we will apply our simple result and the observations above to two examples. These models are not difficult to solve, but they illustrate some techniques and ideas that should be useful even in much more intricate examples. 


\subsubsection{A-model correlators for $\mathbb{P}^{4}$.}

Let us start with the canonical GLSM example: $\mathbb{P}^{4}$. This is a one parameter model with $Q=(1,1,1,1,1)$. The equation of motion from eqn.(5) is just $\sigma^{5}=q$, and $\widetilde{H}=5 \sigma^{4}$, yielding

$$
\left\langle\sigma^{a}\right\rangle_{g}=5^{g-1} \sum_{\sigma \mid \sigma^{5}=q} \sigma^{a+4(g-1)} .
$$

The correlators satisfy the selection rule discussed earlier:

$$
\left\langle\sigma^{a}\right\rangle_{g}=0 \text { unless } a+4(g-1)=5 n \text { for some integer } n
$$

in which case

$$
\left\langle\sigma^{5 n+4(1-g)}\right\rangle_{g}=5^{g} q^{n}
$$

The all-genus correlation function is given by

$$
\left\langle\sigma^{a}\right\rangle=\sum_{g \geq 0} \lambda^{2 g-2}\left\langle\sigma^{a}\right\rangle_{g}
$$

Evaluating this for $0 \leq a \leq 4$, we find

$$
\begin{aligned}
\left\langle\sigma^{a}\right\rangle & =\frac{5\left(5 q \lambda^{2}\right)^{a}}{1-5^{5} q^{4} \lambda^{10}}, a=0, \ldots, 3 \\
\left\langle\sigma^{4}\right\rangle & =\frac{\lambda^{-2}}{1-5^{5} q^{4} \lambda^{10}}
\end{aligned}
$$

The intriguing pole at $q^{4} \lambda^{10}=5^{-5}$ agrees with the findings of [16]. The interpretation of this pole is far from clear. While we might expect such a pole in a topological string theory, where it could be a manifestation of non-perturbative effects in $\lambda$, we have not coupled the model to $d=2$ gravity, and thus any string-based interpretation does not seem appropriate.

Finally, we can use the quantum restriction formula to compute the unique $A$-model correlator on the quintic in $\mathbb{P}^{4}$. The anti-canonical divisor corresponds to $-K=5 \sigma$, and we find

$$
\left\langle\left\langle\sigma^{3}\right\rangle\right\rangle_{g=0}=\operatorname{Tr} \frac{\sigma^{3}}{5 \sigma^{4}} \frac{5 \sigma}{1+5 \sigma}=\operatorname{Tr} \frac{1}{1+(5 \sigma)^{5}}=\frac{5}{1+5^{5} q}
$$




\subsubsection{A Two Parameter Example}

This is another example that has been studied in detail in [9]. This GLSM corresponds to the toric variety obtained by resolving the curve of $\mathbb{Z}_{2}$ singularities in the weighted projective space $\mathbb{P}_{1,1,2,2,2}^{4}$. The GLSM has $n=6, r=2$ and charges

$$
Q=\left(\begin{array}{cccccc}
0 & 0 & 1 & 1 & 1 & 1 \\
1 & 1 & 0 & 0 & 0 & -2
\end{array}\right)
$$

Obviously, $\Delta=4$, and $\widetilde{H}=8 \sigma_{1}^{3} \sigma_{2}$. Letting $\sigma_{2}=\omega \sigma_{1}$, the equations of motion $d \widetilde{W}_{\text {eff }}=0$ may be written as

$$
\sigma_{1}^{4}=\frac{q_{1}}{1-2 \omega}
$$

and

$$
P(\omega)=\omega^{2}-q_{2}(1-2 \omega)^{2}=0 .
$$

The selection rule implies that $\left\langle\sigma_{1}^{a} \sigma_{2}^{b}\right\rangle_{g}$ is zero unless $a+b=4(m+1)$, and if $m \geq 0$, we have

$$
\left\langle\sigma_{1}^{4(m+1)-b} \sigma_{2}^{b}\right\rangle=\sum_{g \geq 0} \operatorname{Tr}\left[\sigma_{1}^{4(m+g)} \omega^{b+g-1}\left(8 \lambda^{2}\right)^{g-1}\right],
$$

which we can reduce to a trace on the roots of $P(\omega)$, denoted by $\operatorname{Tr}^{\prime}$ :

$$
\left\langle\sigma_{1}^{4(m+1)-b} \sigma_{2}^{b}\right\rangle=\frac{q_{1}^{m}}{2 \lambda^{2}\left(1-\left(8 \lambda^{2} q_{1}\right)^{2} q_{2}\right)} \operatorname{Tr}^{\prime}\left[\frac{\omega^{b-1}\left(1+\left(8 \lambda^{2} q_{1}-2\right) \omega\right)}{(1-2 \omega)^{m+1}}\right]
$$

Again, we observe the interesting $\lambda$-dependent pole.

At genus zero the above expression simplifies to

$$
\left\langle\sigma_{1}^{4(m+1)-b} \sigma_{2}^{b}\right\rangle_{g=0}=\frac{q_{1}^{m}}{2} \operatorname{Tr}^{\prime} \frac{\omega^{b-1}}{(1-2 \omega)^{m}} .
$$

We can again use the quantum restriction formula to compute correlators on the anticanonical hypersurface. We have $-K=4 \sigma_{1}$, and

$$
\left\langle\left\langle\sigma_{1}^{3-j} \sigma_{2}^{j}\right\rangle\right\rangle_{g=0}=4\left\langle\frac{\sigma^{4-j} \sigma_{2}^{j}}{1+\left(4 \sigma_{1}\right)^{4}}\right\rangle_{g=0}=2 \operatorname{Tr}^{\prime} \frac{\omega^{j-1}(1-2 \omega)}{1-4^{4} q_{1}-2 \omega} .
$$

In this and other two-parameter models it is convenient to rewrite the $\operatorname{Tr}^{\prime}$ as a contour 
integral:

$$
\operatorname{Tr}^{\prime} f(\omega)=\sum_{\hat{\omega} \mid P(\hat{\omega})=0} \oint_{C(\hat{\omega})} \frac{d \omega}{2 \pi i} \frac{f(\omega) P^{\prime}(\omega)}{P(\omega)}
$$

where $C(\hat{\omega})$ is a small contour about $\omega=\hat{\omega}$. This form makes it easy to evaluate the traces. In the case of more than two parameters, more sophisticated residue techniques may be applied $[11,17]$. Applying this to the case at hand,

$$
\left\langle\left\langle\sigma_{1}^{3-j} \sigma_{2}^{j}\right\rangle\right\rangle_{g=0}=\sum_{\hat{\omega} \mid P(\hat{\omega})=0} \oint_{C(\hat{\omega})} \frac{d \omega}{2 \pi i} \frac{4 \omega^{j}}{\left(1-4^{4} q_{1}-2 \omega\right) P(\omega)} .
$$

Pulling the contour off the roots of $P(\omega)$, the correlators are written as

$$
\left\langle\left\langle\sigma_{1}^{3-j} \sigma_{2}^{j}\right\rangle\right\rangle_{g=0}=\left.2 \frac{\omega^{j}}{P(\omega)}\right|_{\omega=\frac{1-4^{4} q_{1}}{2}}+\left.2 \operatorname{Res}\left\{\frac{\omega^{j}}{\left(\omega-\frac{1-4^{4} q_{1}}{2}\right) P(\omega)}\right\}\right|_{\omega=\infty} .
$$

Straightforward algebra yields

$$
\begin{aligned}
\left\langle\left\langle\sigma_{1}^{3}\right\rangle\right\rangle_{g=0} & =\frac{8}{D} \\
\left\langle\left\langle\sigma_{1}^{2} \sigma_{2}\right\rangle\right\rangle_{g=0} & =\frac{4\left(1-2^{8} q_{1}\right)}{D} \\
\left\langle\left\langle\sigma_{1} \sigma_{2}^{2}\right\rangle\right\rangle_{g=0} & =\frac{8 q_{2}\left(2^{9} q_{1}-1\right)}{\left(1-4 q_{2}\right) D} \\
\left\langle\left\langle\sigma_{2}^{3}\right\rangle\right\rangle_{g=0} & =\frac{4 q_{2}\left(1+4 q_{2}-2^{8} q_{1}-3072 q_{1} q_{2}\right)}{\left(1-4 q_{2}\right)^{2} D}
\end{aligned}
$$

and $D=\left(1-2^{8} q_{1}\right)^{2}-2^{18} q_{1}^{2} q_{2}$. This reproduces the results of eqn.(4.28) of [9]. ${ }^{4}$

\section{A Non-Compact Example}

Having examined the properties of models with a Non-Geometric Phase, we now turn to models where Higgs vacua are present in every phase. Sadly, this means that with the current technology we will need to restrict attention to genus zero correlators, but, nevertheless, we will be able to uncover some surprises.

We will work with the example studied at length in [10]. This GLSM has $n=5, r=2$

\footnotetext{
${ }^{4}$ Our expression corrects a sign error in the last correlator in eqn.(4.28) of [9].
} 


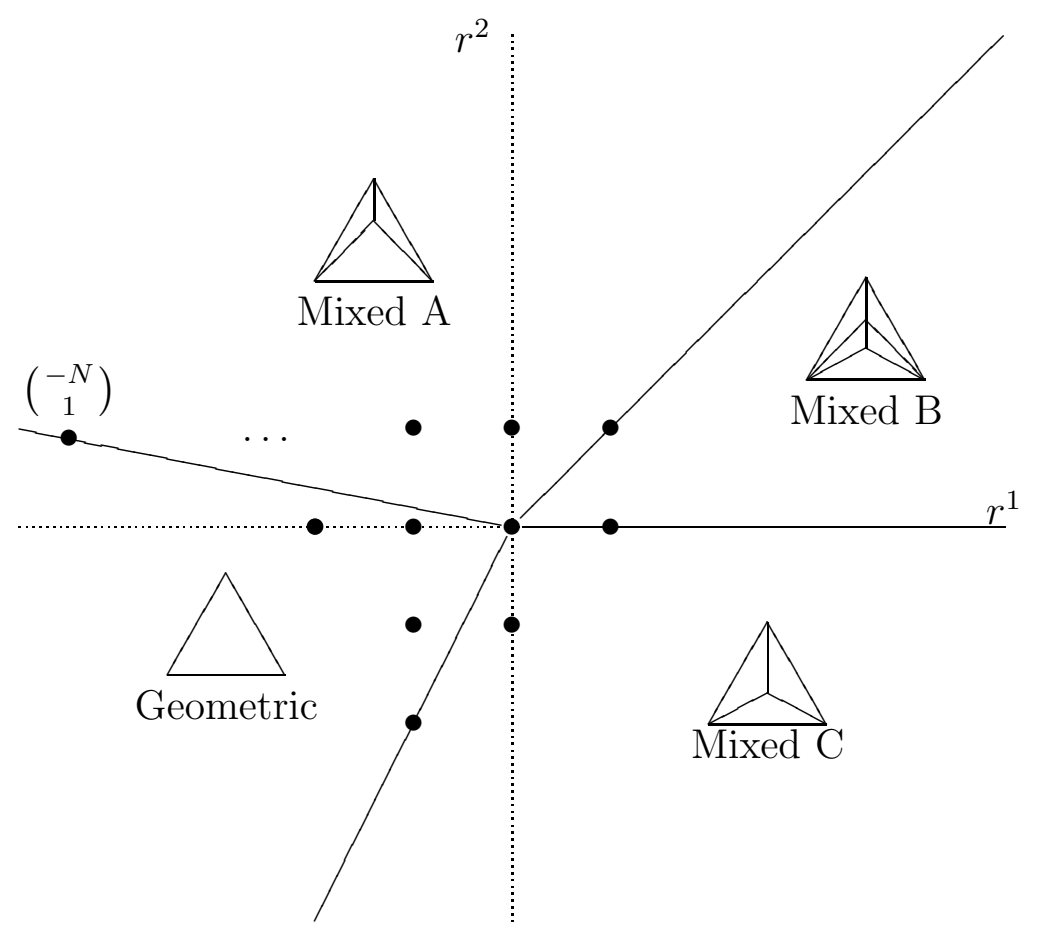

Figure 1: Phases of the GLSM.

and charges

$$
Q=\left(\begin{array}{ccccc}
1 & 1 & 1 & -N & -1 \\
0 & 0 & 1 & 1 & -2
\end{array}\right) .
$$

There are four classical phases, each corresponding to a triangulation of a toric fan. The fan without any subdivisions is an orbifold, $\mathbb{C}^{3} / \mathbb{Z}_{(2 N+1)(2,2,1)}$, the partially subdivided fans correspond to partial resolutions, and the completely subdivided fan is the smooth phase. These phases are depicted in Fig. (II). We will assume $N>2$, and we have labelled the phases according to the Geometric-Mixed terminology defined above. This model has a continuous Coulomb branch which emerges for small $\left|q_{1}\right|$ and $q_{2}=1 / 4$, and, naively, one would expect that some observables in the TFT will be sensitive to this singularity.

The $A$-model correlators of interest are the $Y_{a, b}=\left\langle\sigma_{1}^{a} \sigma_{2}^{b}\right\rangle_{g=0}$. The ghost number selection rule requires that for a non-zero correlator $a+b=3+(2-N) n$. In [10], we were able to compute the $Y_{3+(2-N) n-b, b}$ correlators for $n<0$ by summing the instantons in the Geometric 
Phase, and we found that these correlators could be put into a "Coulomb" form: ${ }^{5}$

$$
Y_{3+(2-N) n-b, b}^{\text {Geometric }}=q_{1}^{n} \operatorname{Tr}^{\prime} \frac{\omega^{b} s(\omega)^{n}}{3 N+1+2 N \omega},
$$

where $\operatorname{Tr}^{\prime}$ is to be taken over the roots of

$$
P(\omega)=(1+\omega)(-N+\omega)-q_{2}(1+2 \omega)^{2},
$$

and $s(\omega)$ is given by

$$
s(\omega)=\frac{(-1-2 \omega)(-N+\omega)^{N}}{1+\omega} .
$$

For later convenience, we will re-write these as a contour integral:

$$
Y_{3+(2-N) n-b, b}=\sum_{\hat{\omega}=\omega_{+}, \omega_{-}} \oint_{C(\hat{\omega})} \frac{d \omega}{2 \pi i} \frac{\omega^{b} s(\omega)^{n}}{(1+2 \omega) P(\omega)},
$$

where $C(\hat{\omega})$ is a small contour about $\omega=\hat{\omega}$, and $\omega_{ \pm}$are the roots of $P(\omega)$.

The computation of the $n=0$ correlators is complicated by the non-compactness of the orbifold, and in [10] we circumvented that problem by using the quantum cohomology relations to determine the $Y_{3-b, b}$. As we saw above, these relations are powerful, and it is easy to show [10] that if the $Y_{3-b, b}$ are determined from the $Y_{3+(2-N) n-b, b}$ by the relations, they must be of given by eqn. (42) with $n=0$. Upon computing the trace, one finds that the $Y_{3-b, b}$ so determined are sensitive to the $q_{2}=1 / 4$ singularity. As we will show below, our basic assumption was incorrect. The quantum cohomology relations simply do not hold!

Even without further computations, there are several reasons to suspect the validity of this result. First, the $Y_{3-b, b}$ are independent of $q_{1}$ and thus, if they are sensitive to the $q_{2}=1 / 4$ singularity at small $\left|q_{1}\right|$, they are equally singular at $q_{2}=1 / 4$ for arbitrary $q_{1}$. However, we are hard pressed to explain the singularity at large $\left|q_{1}\right|$ and $q_{2}=1 / 4$. After all, this is deep in the weakly coupled regime of the Geometric Phase, where a classical analysis is reliable and does not reveal any singularities. In addition, we know that the gauge instantons are labelled by sets of integers $\mathbf{n} \in\left(\mathbb{R}^{d}\right)^{\vee}$, and each such instanton contributes a term $Y^{\mathbf{n}} \prod_{a} q_{a}^{n_{a}}$ to the correlator. In a particular phase $\mathcal{K}_{V}$, the instanton numbers corresponding

\footnotetext{
${ }^{5}$ We call this the "Coulomb" form because it is the answer that one would get by naive application of eqn.(11).
} 
to non-zero $Y^{\mathbf{n}}$ must lie in the dual cone defined by

$$
\mathcal{K}_{V}^{\vee}=\left\{\mathbf{n} \in\left(\mathbb{R}^{d}\right)^{\vee} \mid\langle\mathbf{n}, \mathbf{r}\rangle \geq 0 \quad \forall \quad \mathbf{r} \in \mathcal{K}_{V}\right\}
$$

It is easy to see that in the Geometric Phase the only instantons that can contribute to $Y_{3-b, b}$ have $\mathbf{n}=0$. Hence, one would expect $Y_{3-b, b}$ to be constants, and any $q_{2}$ dependence, let alone a singular one, is strange indeed. This would seem to indicate that the quantum cohomology relations are violated whenever the $Y_{3-b, b}$ correlators are involved.

To explore this further, let us now work out the correlators in one of the Mixed Phases. We will choose the phase $A$, but the computation may be easily repeated for other phases. First, let us compute the contribution from the Coulomb vacua. For this model, $\widetilde{H}$ is given by

$$
\widetilde{H}=(N-2) \sigma_{1}^{2}\left((3 N+1) \sigma_{1}+2 N \sigma_{2}\right)
$$

and the equations of motion that follow from $d \widetilde{W}_{\text {eff }}=0$ are

$$
\begin{aligned}
\left(\sigma_{1}+\sigma_{2}\right)\left(\sigma_{2}-N \sigma_{1}\right) & =q_{2}\left(\sigma_{1}+2 \sigma_{2}\right)^{2} \\
\sigma_{1}^{2}\left(\sigma_{1}+\sigma_{2}\right) & =-q_{1}\left(\sigma_{2}-N \sigma_{1}\right)^{N}\left(\sigma_{1}+2 \sigma_{2}\right)
\end{aligned}
$$

As in the previous section, we may parametrize the solutions by $\sigma_{1}$ and the ratio $\omega=\sigma_{2} / \sigma_{1}$ :

$$
\begin{aligned}
P\left(\omega_{ \pm}\right) & =0 \\
\sigma_{1, \pm ; p} & =\zeta^{p}\left(q_{1} s\left(\omega_{ \pm}\right)\right)^{\frac{1}{2-N}} \\
\sigma_{2, \pm ; p} & =\omega_{ \pm} \sigma_{1, \pm ; p}
\end{aligned}
$$

where $\zeta=e^{\frac{2 \pi i}{N-2}}, p=0, \ldots, N-1$, and $P(\omega)$ and $s(\omega)$ are as in eqns. (43444).

Let us consider the solutions $\left(\sigma_{1, \pm ; p}, \sigma_{2, \pm ; p}\right)$ in the weak coupling limit. Weak coupling in the Mixed Phase A corresponds to

$$
\left|q_{2}\right|^{-N} \gg\left|q_{1}\right| \gg\left|q_{2}\right|,
$$


and in particular, $\left|q_{2}\right| \rightarrow 0$. In this limit the $\sigma$-vacua have a simple structure:

$$
\begin{aligned}
& \omega_{+} \rightarrow-1-\frac{1}{N+1} q_{2}, \\
& \omega_{-} \rightarrow N+\frac{(2 N+1)^{2}}{N+1} q_{2},
\end{aligned}
$$

and hence

$$
\begin{aligned}
& \sigma_{1 ;+}^{2-N} \rightarrow q_{1} q_{2}^{-1}(-N-1)^{N+1} \\
& \sigma_{2 ;-}^{2-N} \rightarrow-q_{1} q_{2}^{N} \frac{(2 N+1)^{2 N+1}}{(N+1)^{N+1}}
\end{aligned}
$$

Thus, we see that in the weak coupling limit of the Mixed phase the $N-2$ "-" critical points of $\widetilde{W}_{\text {eff }}$ have growing $\sigma$ vevs, while the $N-2$ "+" critical points have decreasing $\sigma$ vevs. Thus, only the "-" solutions correspond to actual Coulomb vacua, and their contribution, $Y_{3+(2-N) n-b, b}^{\text {Coulomb }}$, is given by the $\omega_{-}$contribution in eqn.(45):

$$
Y_{3+(2-N) n-b, b}^{\text {Coulomb }}=q_{1}^{n} \oint_{C\left(\omega_{-}\right)} \frac{\omega^{b} s(\omega)^{n}}{(1+2 \omega) P(\omega)}
$$

Next, we consider the Higgs contribution. Unlike the Coulomb computation, which gives the same form regardless of whether $n=0$ or $n<0$, here this distinction makes a crucial difference. First, let us consider the situation when $n<0$. Using the standard toric techniques of Morrison and Plesser, we can perform the instanton sum and evaluate the Higgs branch contribution to the correlators. Performing the requisite toric intersection computations, we reduce the correlators to a single sum:

$$
Y_{3+(2-N) n-b, b}^{\mathrm{Higgs}}=-q_{1}^{n} \sum_{m=-n}^{\infty} \oint_{C(-1)} \frac{d \omega}{2 \pi i} \frac{\omega^{b} s(\omega)^{n} R^{m}}{(1+\omega)(-N+\omega)(-1-2 \omega)}
$$

where

$$
R=q_{2} \frac{(1+2 \omega)^{2}}{(1+\omega)(-N+\omega)}
$$

and $C(-1)$ is a small contour about $\omega=-1: \omega=-1+\epsilon e^{i \theta}$. For uniform convergence we must have

$$
\left|q_{2}\right|<\frac{\epsilon(N+1-\epsilon)}{1+2 \epsilon}
$$


Provided that this condition holds, we can exchange the integral and the sum to obtain

$$
Y_{3+(2-N) n-b, b}^{\mathrm{Higgs}}=q_{1}^{n} \oint_{C(-1)} \frac{\omega^{b} s(\omega)^{n} R^{-n}}{(1+2 \omega) P(\omega)} .
$$

And now comes a pleasant surprise: the condition for convergence ensures that $\omega=\omega_{+}$is enclosed by $C(-1)$, while $\omega=\omega_{-}$remains outside of it, and so, since $R\left(\omega_{ \pm}\right)=1$,

$$
Y_{3+(2-N) n-b, b}^{\mathrm{Higgs}}=q_{1}^{n} \oint_{C\left(\omega_{+}\right)} \frac{d \omega}{2 \pi i} \frac{\omega^{b} s(\omega)^{n}}{(1+2 \omega) P(\omega)},
$$

and for $n<0$ we precisely have the desired form for the correlators:

$$
Y_{a, b}^{\text {Geometric }}=Y_{a, b}^{\mathrm{Higgs}}+Y_{a, b}^{\text {Coulomb }} .
$$

The contribution to the $n=0$ correlators is even more remarkable. The standard manipulation of the instanton sum yields

$$
Y_{3-b, b}^{\mathrm{Higgs}}=Y_{3-b, b}^{0}+\sum_{m=0}^{\infty} \oint_{C(N)} \frac{d \omega}{2 \pi i} \frac{\omega^{b} R^{m}}{(1+\omega)(-N+\omega)(-1-2 \omega)},
$$

with $R$ as above, and $C(N)$ a small contour about $\omega=N: \omega=N+\epsilon e^{i \theta}$. The constants $Y_{3-b, b}^{0}$ parametrize our ignorance of how to compute intersection numbers on a non-compact variety. Presumably, these are computed by an appropriate cohomolgy theory. For uniform convergence we must have

$$
\left|q_{2}\right|<\frac{\epsilon(1-\epsilon)}{1+2 N+\epsilon}
$$

Carrying out the sum, we have

$$
Y_{3-b, b}^{\mathrm{Higgs}}=Y_{3-b, b}^{0}-\oint_{C(N)} \frac{d \omega}{2 \pi i} \frac{\omega^{b}}{(1+2 \omega) P(\omega)} .
$$

The condition for convergence ensures that, this time, $\omega_{-}$is enclosed by $C(N)$, while $\omega_{+}$ remains outside. The crucial overall minus sign means that putting this together with the Coulomb contribution, we find that the $Y_{3-b, b}$ are just constants, as predicted by our discussion above.

A similar analysis may be carried out in the other Mixed Phases. In those phases all of 
the Coulomb vacua are reliable and contribute. For $n<0$ there are no contributions from the gauge instantons of the Higgs branch, while for $n=0$ the instanton sums cancel the Coulomb contribution up to the constants $Y_{3-b, b}^{0}$. It would be interesting to examine these constants in more detail, but whatever they are, the resulting correlators are incompatible with quantum cohomology relations.

One final aspect of this example deserves mention - the disappearance of the semiclassical singularity at $q_{2}=1 / 4$. We know that semi-classical analysis of the Higgs branch in Mixed Phase B or $\mathrm{C}$ shows this singularity. We expect that analysis to be valid for small $\left|q_{1}\right|$. Of course, the discrete Coulomb vacua also exist in this limit, and it is possible that the presence of these additional Coulomb vacua washes out the singularity. It appears that this is so, at least in the topological theory.

\section{Discussion}

We have found a simple algebraic formula for the Coulomb contribution to the $A$-model correlators in toric GLSMs. We hope to have convinced the reader that this expression is conceptually satisfying and computationally useful. We will now conclude with an outlook on some interesting questions that remain.

\subsection{Some Observations on the Coulomb Vacua and the GLSM}

Our work is a simple application of the general principle of localization in TFTs. It has been known for a long time that in the Geometric Phases the path integral localizes onto the gauge instantons. We have merely extended this result to the Mixed and Non-Geometric Phases. In models with a Non-Geometric Phase our result gives a surprisingly complete form for the $A$-model correlators. In models without such a phase we are restricted to genus zero by our inability to compute the contribution from the Higgs vacua.

Perhaps the most surprising finding of our work is that for models without a NonGeometric Phase the quantum cohomology relations may fail for a subset of the correlators which, in some Mixed Phase, receive contributions from both Higgs and Coulomb vacua. There are two simple "proofs" of quantum cohomology relations: the first is a Geometric Phase analysis of the intersection numbers on the gauge instanton moduli spaces $[9,10]$, and the second is a Non-Geometric Phase analysis given above. The first argument is subtle 
when the phase corresponds to a non-compact variety, and the second does not apply in the absence of a Non-Geometric Phase. The example of the last section illustrates that these problems are manifestations of the same failure of the quantum cohomology relations in different phases.

We have not addressed computations of the correlators in phases where discrete HiggsCoulomb vacua are present. It would be interesting to understand the contributions from these vacua. This exercise may well provide some new insights into the more mysterious aspects of GLSM physics, and it may provide us with another set of phases where the computation of the correlators is made tractable.

Finally, we have derived our results in the context of traditional GLSMs. It would be interesting to extend our treatment to the recently much-discussed GLSMs corresponding to supermanifolds.

\subsection{A Pure Landau-Ginzburg Description?}

The contribution to the $A$-model correlators bears a striking resemblance to Vafa's result on correlators in topological Landau-Ginzburg models [11]. Vafa studied a topological LandauGinzburg model with a superpotential $W(X)$, and he showed that the correlators are given by

$$
\langle F(x)\rangle=\operatorname{Tr} F(x) H^{g-1}
$$

where the trace is taken over the critical points of $W$, and each contribution is weighted by the Hessian of $W, H$, evaluated at that point.

While it is certainly not true that the GLSM model correlators are computed by a Landau-Ginzburg theory with $\Sigma_{a}$ as the fields and $\widetilde{W}_{\text {eff }}$ as the superpotential, because of the remarkable similarity between eqn.(11) and eqn.(63), it is natural to wonder if some LandauGinzburg theory computes the same correlators. It is easy to see that this is possible for at least some models. For example, a Landau-Ginzburg theory of a single field $\Sigma$ and superpotential $W=\frac{1}{6} \Sigma^{6}-q \Sigma$ has the equation of motion $\sigma^{5}=q$ and Hessian of $5 \sigma^{4}$. Hence, according to eqn.(63), this model will compute the same correlators as the GLSM with target space $\mathbb{P}^{4}$.

It is clear that, in general, the requisite Landau-Ginzburg model will be much more complicated. After all, the theories studied by Vafa can be constructed as relevant deformations of a free theory with an ultraviolet $R$-symmetry with charges $Q_{+}(X)=Q_{-}(X)=0$, and 
all of the solutions to the classical equations of motion $d W(x)=0$ are on the same footing. It is tempting to suggest that the method of Hori and Vafa [18], which relies on dualizing the matter fields, yields this Landau-Ginzburg description. It would be interesting to check whether this is the case.

\subsection{Coupling to Topological Gravity}

Another direction to pursue is to couple the model to topological gravity in the spirit of [16]. The resulting theory would be an interesting topological string theory, where perhaps the $\lambda$ dependent poles we have found would find a natural interpretation. Furthermore, this model would, in principle, compute a much larger subset of non-trivial Gromov-Witten invariants. Hopefully, the simplicity of our result for the correlators would persist to some extent in the string theory.

\section{Acknowledgments}

It is a pleasure to thank C. Haase, E. Katz, G. Moore, and S. Rinke for useful comments and conversations. M.R.P. would like to thank the Perimeter Institute and the Theoretical High Energy Physics group at Rutgers University for hospitality while some of this work was completed. This article is based upon work supported in part by the National Science Foundation under Grants DMS-0074072 and DMS-0301476. Any opinions, findings, and conclusions or recommendations expressed in this article are those of the authors and do not necessarily reflect the views of the National Science Foundation.

\section{References}

[1] E. Witten. Topological sigma models. Commun. Math. Phys., 118:411, 1988.

[2] E. Witten. Mirror manifolds and topological field theory. 1991, arXiv:hep-th/9112056.

[3] P. Candelas, X.C. de la Ossa, P.S. Green, and L. Parkes. A pair of Calabi-Yau manifolds as an exactly soluble superconformal theory. Nucl. Phys., B359:21-74, 1991.

[4] P. Candelas, X.C. de la Ossa, A. Font, S. Katz, and D.R. Morrison. Mirror symmetry for two parameter models. 1. Nucl. Phys., B416:481-538, 1994, arXiv:hep-th/9308083. 
[5] P. Candelas, A. Font, S. Katz, and D.R. Morrison. Mirror symmetry for two parameter models. 2. Nucl. Phys., B429:626-674, 1994, arXiv:hep-th/9403187.

[6] S. Hosono, A. Klemm, S. Theisen, and S.-T. Yau. Mirror symmetry, mirror map and applications to calabi-yau hypersurfaces. Commun. Math. Phys., 167:301-350, 1995, arXiv:hep-th/9308122,

[7] S. Hosono, A. Klemm, S. Theisen, and S.-T. Yau. Mirror symmetry, mirror map and applications to complete intersection calabi-yau spaces. Nucl. Phys. B, 433:501-554, 1995, arXiv:hep-th/9406055.

[8] E. Witten. Phases of $N=2$ theories in two dimensions. Nucl. Phys., B403:159-222, 1993, arXiv:hep-th/9301042.

[9] D.R. Morrison and M.R. Plesser. Summing the instantons: Quantum cohomology and mirror symmetry in toric varieties. Nucl. Phys., B440:279-354, 1995, arXiv:hep-th/9412236.

[10] I.V. Melnikov and M.R. Plesser. The Coulomb branch in gauged linear sigma models. Journal of High Energy Physics, 2005(06):013, 2005, arXiv:hep-th/0501238.

[11] Cumrun Vafa. Topological Landau-Ginzburg models. Mod. Phys. Lett., A6:337-346, 1991.

[12] A. Schwarz and O. Zaboronsky. Supersymmetry and localization. Commun. Math. Phys., 183:463-476, 1997, ArXiv:hep-th/9511112.

[13] M. Blau and G. Thompson. Localization and diagonalization: A review of functional integral techniques for low dimensional gauge theories and topological field theories. $J$. Math. Phys., 36:2192-2236, 1995, ArXiv:hep-th/9501075.

[14] S. Cordes, G.W. Moore, and S. Ramgoolam. Lectures on 2-d Yang-Mills theory, equivariant cohomology and topological field theories. Nucl. Phys. Proc. Suppl., 41:184-244, 1995, ArXiv:hep-th/9411210.

[15] P.S. Aspinwall, B.R. Greene, and D. R. Morrison. Calabi-Yau moduli space, mirror manifolds and space-time topology change in stringy geometry. Nucl. Phys., B416:414480, 1994, arXiv:hep-th/9606112. 
[16] E. Witten. On the structure of the topological phase of two-dimensional gravity. Nucl. Phys., B340:281-332, 1990.

[17] Phillip Griffiths and Joseph Harris. Principles of Algebraic Geometry. Wiley Classics Library. John Wiley \& Sons Inc., New York, 1994.

[18] K. Hori and C. Vafa. Mirror symmetry. 2000, arXiv:hep-th/0002222. 